\title{
ANÁLISE PROBABILÍSTICA DA CAPACIDADE MECÂNICA DE SAPATAS DE CONCRETO ARMADO EM SOLO CORROSIVO
}

\author{
FELIX, EMERSON FELIPE \\ Doutorando em Eng. Civil/Estruturas \\ USP/SET/EESC \\ São Carlos/SP; Brasil \\ emerson.felipe.felix@gmail.com
}

\author{
AMORIM, DANILO MEIRA \\ Graduando em Eng. Civil \\ Centro Universitário Estácio de Ribeirão Preto \\ Ribeirão Preto/SP; Brasil \\ danilomeira.amorim@gmail.com
}

\section{RESUMO}

Partindo do princípio da importância da fundação na execução de um projeto, visto que ela é responsável por receber e distribuir todas as cargas da superestrutura para o terreno natural, o presente trabalho tem por objetivo analisar a capacidade mecânica de sapatas de concreto armado executadas em solo corrosivo por meio de simulações numéricas via Monte Carlo. O modelo mecânico-probabilístico obtido via Monte Carlo foi implementado em linguagem Fortran. Os resultados obtidos demonstram que a resistência à compressão do concreto possui influência secundária diante dos demais parâmetros, e mais, que a depender do cobrimento e do NSPT a capacidade de carga da sapata pode ser drasticamente reduzida. Por fim, destaca-se que os resultados obtidos neste trabalho, apontam a aplicabilidade da técnica de simulação para o estudo da capacidade de carga de elementos de fundações.

Palavras-chave: sapata de concreto, capacidade mecânica, solo corrosivo, simulação de Monte Carlo.

\section{ABSTRACT}

Assuming the importance of the foundation in the execution of a project, since it is responsible for receiving and distributing all loads of the superstructure to the natural terrain, the present work aims to analyze the mechanical capacity reinforced concrete spread footing executed in corrosive soil through numerical simulations via Monte Carlo. The mechanical-probabilistic model obtained via Monte Carlo was implemented in Fortran. The results show that the compressive strength of concrete has a secondary influence on the other parameters, and more, that depending on the covering and the NSPT the load capacity of the spread footing can be drastically reduced. Finally, it is noteworthy that the results obtained in this work indicate the applicability of the simulation technique for the study of the load capacity of foundation elements.

Keywords: concrete spread footing, mechanical capacity, corrosive soil, Monte Carlo simulation.

\section{INTRODUÇÃO}

Dentre os mais diversos tipos de obra inseridos no setor da construção civil, as estrutura têm grande importância e diversas classificações, às quais podem ser subdivididas em: superestruturas, referente aos elementos estruturas que estão acima da linha de solo, por exemplo, elementos de pilares, vigas e lajes, e os elementos de fundações ou infraestrutura, que são responsáveis por receber toda a carga da superestrutura e distribuí-la de forma contínua para o solo.

De acordo com Alonso (2011), dependendo do tipo de edificação, os elementos de fundações geralmente se encontram abaixo do nível do solo, como exemplo, no Brasil, as fundações mais utilizadas são as fundações superficiais (blocos e sapatas), e as profundas (estacas e tubulões). Essa classificação é feita de acordo com a transmissibilidade das tensões da superestrutura para o solo, assim as fundações superficiais, são aquelas em que as cargas são transmitidas ao terreno diretamente pela base, e as profundas, são quando as tensões são também transmitidas pelo fuste, ou pela lateral do elemento de fundação.

De modo geral, um elemento de fundação deve ser dimensionado para que as tensões de compressão geradas sejam resistidas pelo próprio concreto, fazendo com que fatores condicionantes como a capacidade de carga do solo, os critérios 
de ruptura e campos de deslocamentos (recalques) também sejam requisitos de análise, a fim de garantir um projeto econômico e seguro. Velloso e Lopes (2010) destacam que uma análise rigorosa dos fatores condicionantes, anteriormente relatados, deve ser realizada ainda na fase de projeto, visto que mudanças na configuração geométrica, seja por falhas executivas no período de construção ou manifestações patológicas ao longo da vida útil, podem provocar mudanças drásticas no solo ocasionando sérios riscos e prejuízos a superestrutura.

A ocorrência de manifestações patológicas em construções e edificações é um problema observado em todas as regiões do Brasil e, no mundo, gerando conflitos de interesse, onde as verdadeiras causas acabam por muitas vezes sendo desconhecidas (SILVA JUNIOR, 2008). De acordo com Logeais (1982), quando o assunto se refere a manifestações patológicas em fundações, os maiores problemas ocorrem devido ao desconhecimento das características do solo, onde o dimensionamento é realizado sob a consideração de parâmetros de projeto coletados por ensaios de sondagens realizados de maneira errônea.

Assim, quando o solo não é bem caracterizado diversos problemas podem aparecer ao longo da vida útil de um elemento de fundação. As manifestações patológicas em fundações caracterizam o mau desempenho do elemento estrutural sendo observadas na maioria dos casos pela deterioração dos materiais constituintes, por deformações excessivas, perda de cobrimento mínimo, corrosão de armaduras, fissuração excessiva e ruptura local e/ou geral (ABMS/ABEF, 2016).

A corrosão de armaduras, é a manifestação patológica de maior incidência em estruturas de concreto. No Brasil, por exemplo, a corrosão possuí um índice de ocorrência que varia de 14 a $64 \%$ a depender da região de análise (CARMONA; MAREGA, 1988; DAL MOLIN, 1988; ANDRADE, 1992). A corrosão ocorre no concreto armado, quando as barras de aço, imersas na matriz de concreto, perdem a sua camada química protetora (camada passivadora), sendo o mecanismo de despassivação desencadeado pela ação do $\mathrm{CO}^{2}$ (corrosão por carbonatação) e/ou cl- (corrosão por cloretos).

A corrosão é definida como um processo eletroquímico, causado pelas diferenças nas concentrações de íons dissolvidos, de maneira que parte do metal se torna catódica e outra anódica, resultando na perda de volume do material, gerando produtos de corrosão, e consequente redução de capacidade de suporte (MEHTA; MONTEIRO, 2014; GEIKER; POLDER, 2016). Assim, considerando que os elementos de fundações geralmente são dimensionados de modo que a sua configuração geométrica transmita cargas contínuas e bem distribuídas ao solo, se a geometria é modificada ou os materiais constituintes perdem resistência, as cargas transmitidas serão drasticamente intensificadas, podendo levar a sobrecarga e possivelmente ruptura do solo, se a sua capacidade de carga for superada.

A capacidade de carga do solo segundo a NBR 6122 (ABNT, 2010), pode ser determinada através ensaios de provas de carga sobre placas ou pela utilização de formulações analíticas ou ábacos existentes na literatura, por meio de métodos teóricos (TERZAGHI, 1943; MEYERHOF, 1963; VÉSIC, 1974), métodos empíricos e semi-empíricos.

Devido aos diversos métodos e ferramentas que são utilizados atualmente para a verificação de desempenho e análise da capacidade de carga de um solo (ALONSO, 1991; BARATA, 1984; VELLOSO \& LOPES, 2004) e pelas recomendações prescritas na NBR 6122 (ABNT, 2010), os resultados obtidos são dados sempre a favor da segurança, porém, cada um destes métodos é validado para diferentes condições, que muitas vezes não atendem ao tipo de solo em que se pretende realizar a implantação do elemento de fundação. Ademais, acrescenta-se que existe uma variabilidade dos parâmetros obtidos em ensaios de sondagem, que em muitos casos, por motivos de economia ou da não existência de mão de obra qualificada, há a utilização de um único tipo de ensaio, que no Brasil é o SPT (Standard Penetration Test).

Diante do exposto, o presente trabalho tem como objetivo apresentar uma metodologia de análise mecânico-probabilística da capacidade mecânica de sapatas de concreto armado via simulações de Monte Carlo. Para a determinação das equações de estado limite, foram utilizadas as formulações propostas por Terzaghi (1943) e Vésic (1974), a fim de analisar a capacidade de carga do solo, conjuntamente com método dimensionamento de bielas e tirantes, para analisar a capacidade resistente dos elementos de fundação.

\section{MÉTODOS TÉORICOS PARA DETERMINAÇÃO DA CAPACIDADE DE CARGA}

Na sequência são apresentados os métodos téoricos utilizados neste trabalho, a fim de determinar a capacidade e carga de fundações superficiais do tipo sapata de concreto. 


\subsection{Formulação clássica de Terzaghi (1943)}

Para desenvolvimento de seu estudo, Terzagui considerou as seguintes hipóteses:

- O comprimento L (medida perpendicular ao plano da fundação, indentificado na Figura 1) do elemento de fundação deve ser bem maior que a largura B (medida paralela ao elemento de fundação identifacado na Figura 1) $(\mathrm{L} / \mathrm{B}>5)$, ;

- A largura B da sapata deve ser maior que a profundidade h, de assentamento da base da sapata (h <= B);

- O maciço deve apresentar ruptura generalizada, sendo essa, caracterizada por solos muito compactos ou consistentes, apresentando uma superficie de deslizamento bem definida e tendência de levantamento do solo adjacente a fundação. De acordo com Alonso (2011), nestes casos a ruptura é brusca e catastrófica.

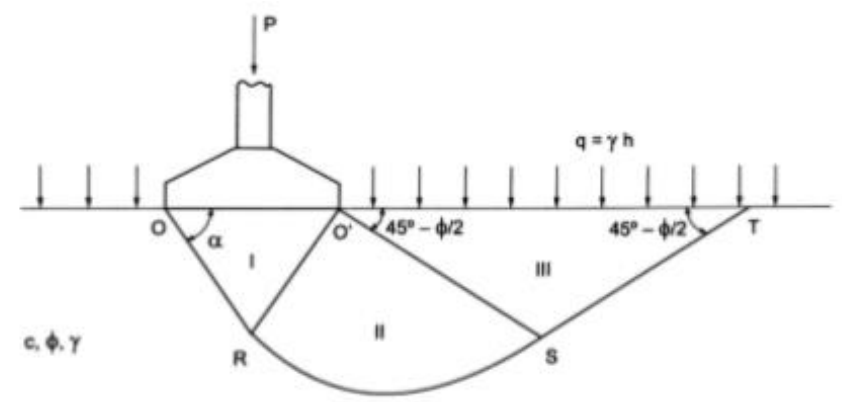

Figura 1: Superfície potencial de ruptura.

Fonte: Cintra et al. (2003).

A Figura 1 apresenta uma esquematização da superfície potencial de ruptura do solo, que é composta por três diferentes regiões:

- Região I: onde a superfície de ruptura apresenta um trecho reto;

- Região II: onde a superfície potencial de ruptura apresenta a forma de uma espiral logarítmica;

- Região III: onde a superfície de ruptura apresenta um trecho reto e uma cunha.

Segundo Campos (2015), de acordo com a teoria de Terzaghi (1943), a ruptura do solo ocorre por puncionamento, caracterizando-se pelo deslocamento vertical abaixo do elemento de fundação, fazendo com que surjam empuxos laterais de terra, sendo transmitidos de região a região, fazendo com que a superfície de ruptura seja mobilizada.

Desse modo, a capacidade de carga proposta por Terzagui (1943), pode ser calculada pela Equação 1, onde os fatores de forma $\left(\mathrm{S}_{\mathrm{C}}, \mathrm{S}_{\mathrm{q}}\right.$ e $\left.\mathrm{S}_{\gamma}\right)$ e de capacidade de carga $\left(\mathrm{N}_{\mathrm{C}}, \mathrm{N}_{\mathrm{q}}\right.$ e $\left.\mathrm{N}_{\gamma}\right)$, podem ser obitdos de acordo com a Tabela 1 e o ábaco apresentado na Figura 2, respectivamente.

$$
\sigma_{r}=c N_{c} S_{c}+\mathrm{q} N_{q} S_{a}+0,5 \gamma N_{\gamma} S_{y}
$$

(Equação 1)

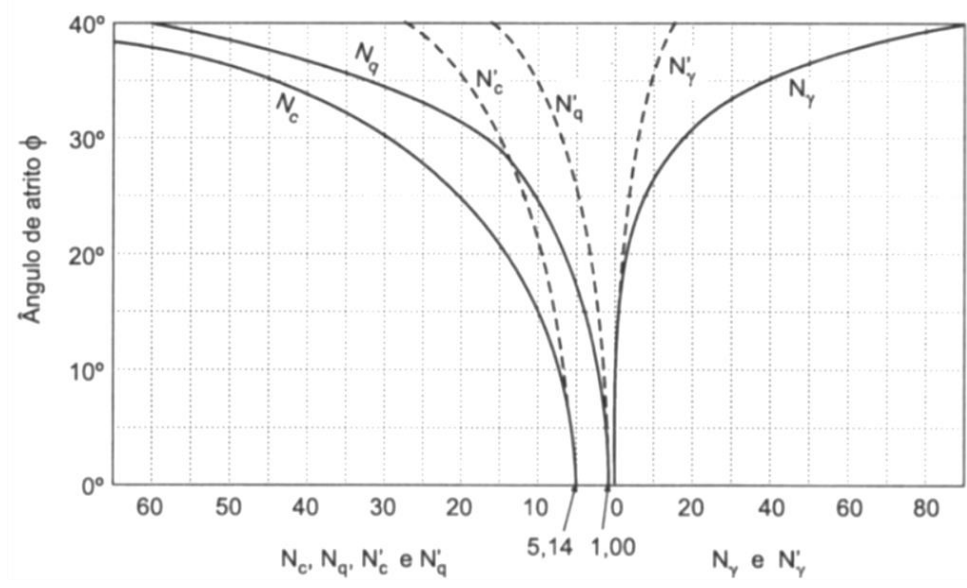

Figura 2: Superfície potencial de ruptura.

Fonte: Cintra et al. (2003). 
Tabela 1 - Fatores de forma.

\begin{tabular}{c|c|c|c}
\hline \multirow{2}{*}{ Forma da sapata } & \multicolumn{3}{|c}{ Fatores de forma } \\
\cline { 2 - 4 } & $\mathrm{S}_{\mathrm{C}}$ & $\mathrm{S}_{\mathrm{q}}$ & $\mathrm{S}_{\gamma}$ \\
\hline Corrida & 1,0 & 1,0 & 1,0 \\
\hline Quadrada & 1,3 & 1,0 & 0,8 \\
\hline Circular & 1,3 & 1,0 & 0,6 \\
\hline
\end{tabular}

\subsection{Formulação de Vésic (1974)}

A metodologia proposta por Vésic, cuja versão mais recente data de 1974, apresenta uma série de refinamentos que não foram considerados na formulação clássica de Terzaghi.

A formulação desenvolvida consiste basicamente na incorporação e modificações de alguns parâmetros e variáveis utilizadas na metodologia de Terzaghi (1943) e outras formulações decorrentes desta, por exemplo, Meyerhof (1963) e Hansen (1970). Assim, em relação ao método de Terzaghi (1943), já apresentado neste trabalho, o cálculo da capacidade de carga desenvolvido por Vésic (1974), leva em consideração a introdução de fatores que expressam:

- Influência da profundidade de assentamento da fundação $\left(d_{C}, d_{q}\right.$ e $\left.d_{\gamma}\right)$;

- Influência da inclinação da carga aplicada em relação à normal ao plano do elemento de fundação $\left(i_{C}, i_{q}\right.$ e $\left.i_{\gamma}\right)$;

- Influência da inclinação do terreno adjacente ao elemento de fundação $\left(g_{C}, g_{q}\right.$ e $\left.g_{\gamma}\right)$;

- Influência da inclinação da base do elemento de fundação em relação ao plano horizontal $\left(b_{C}, b_{q} \mathrm{e} \mathrm{b}_{\gamma}\right)$.

Desse modo, a capacidade de carga proposta por Vésic (1943), pode ser calculada conforme Equação 2, onde os fatores de forma $\left(S_{C}, S_{q}\right.$ e $\left.S_{\gamma}\right)$ e de capacidade de carga $\left(N_{C}, N_{q}\right.$ e $\left.N_{\gamma}\right)$, podem ser obtidos ainda por intermédio da Tabela 1 e do ábaco apresentado na Figura 2. Ademais, como medidas para os cálculos, deve-se adotar $B$ como sendo a menor dimensão da sapata, $q$ é a tensão efetiva na cota de assentamento, $\left(d_{C}, d_{q}\right.$ e $\left.d_{\gamma}\right)$ são os fatores de profundidade, $\left(i_{C}, i_{q}\right.$ e $\left.i_{\gamma}\right)$ são os fatores de inclinação de carga em relação à base do elemento de fundação, $\left(g_{C}, g_{q}\right.$ e $\left.g_{\gamma}\right)$ são os fatores de inclinação do terreno adjacente à fundação $\mathrm{e}\left(b_{C}, b_{q} \mathrm{e} \mathrm{b}_{\gamma}\right)$ são os fatores de inclinação da fundação em relação à horizontal. Para o cálculo dos fatores de forma de inclinação, que são índices de correção em relação a formulação de Terzaghi (1943), pode-se consultar Alonso (2011).

$$
\begin{gathered}
\rho_{r}=c \cdot N_{c} \cdot S_{c} \cdot d_{c} \cdot i_{c} \cdot g_{c} \cdot b_{c}+\mathrm{q} \cdot N_{q} \cdot S_{q} \cdot d_{q} \cdot g_{q} \cdot i_{q} \cdot b_{q}+ \\
+0,5 \cdot B \cdot \gamma \cdot N_{\gamma} \cdot S_{\gamma} \cdot d_{\gamma} \cdot g_{\gamma} \cdot i_{\gamma} \cdot b_{\gamma}
\end{gathered}
$$

(Equação 2)

\section{METODOLOGIA}

A fim de determinar a probabilidade de uma fundação superficial do tipo sapata entrar em ruína no decorrer de um período de 60 anos (vida útil de projeto), uma métodologia de análise probabilistica via simulação de Monte Carlo foi empregada, a qual é apresentada por meio de um fluxograma, conforme mostra a Figura 3. No fluxograma é possível ver todas as atividades realizadas para que ao final fossem realizadas as análises de perda de capacidade de carga do solo e de resistência do elemento de fundação.

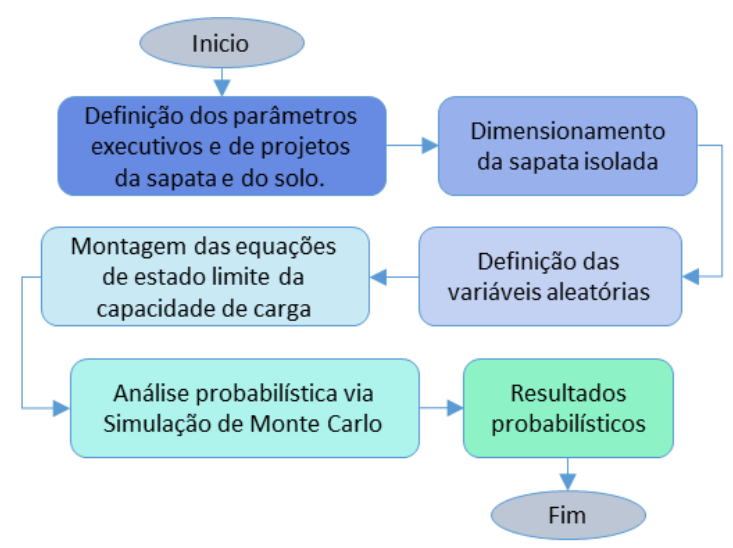

Figura 3: Fluxo de desenvolvimento do trabalho. 
A fim de determinar a probabilidade de uma sapata de concreto armado inserida em solo corrosivo entrar em colapso durante sua vida útil de projeto, período de 60 anos, de acordo com a NBR 6118 (ABNT, 2014), uma abordagem mecânico-probabilística via simulação de Monte Carlo foi considerada neste trabalho, de forma a inserir no processo de modelagem a aleatoriedade dos parâmetros envolvidos no problema. Na sequência são apresentadas as formulações analíticas utilizadas para determinar a despassivação do aço, perda seção útil (capacidade mecânica do elemento estrutural) e perda de capacidade de carga do solo. São apresentadas ainda, o dimensionamento da sapata de concreto analisada e as principais equações que regem o método de simulação de Monte Carlo.

\subsection{Dimensionamento da sapata}

A seguir, são apresentados os parâmetros coletados no dimensionamento de uma sapata isolada rígida de concreto. A sapata dimensionada recebe uma carga permanente $\left(G_{k}\right)$ de $1320 \mathrm{kN}$ e acidental $\left(Q_{k}\right)$ de $570 \mathrm{kN}$. O pilar e seu posicionamento geométrico é apresentado na Figura 4. Considera-se o cimento Portland utilizado foi o CP III.

Dados para o dimensionamento:

- Pilar:

- $b_{1}=30 \mathrm{~cm}$ e $b_{2}=80 \mathrm{~cm}$ (dimensões do pilar);

- $f_{c k}=20 \mathrm{Mpa}$ (resistência à compressão do concreto);

- Solo:

- $R_{d, \text { solo }}=0,357 \mathrm{MPa}$ (tensão resistente do solo);

- $\rho_{\text {adm, solo }}=0,255 \mathrm{MPa}$ (tensão admissível do solo);

- Aço:

- $f_{y k}=500 \mathrm{MPa}$ (resistência do aço);

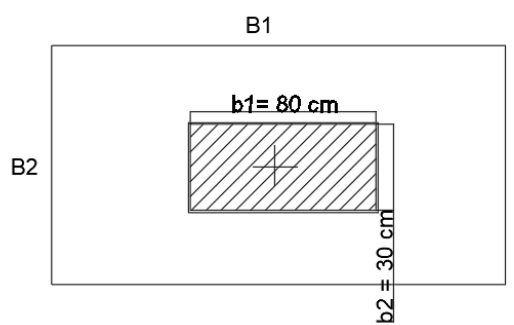

Figura 4: Esquema de representação do pilar e da sapata.

A carga solicitante é dada na Equação 3, e a área da sapata que fará a transmissão da carga do pilar para o solo é dada na Equação 4. Considerando a proporcionalidade das dimensões da sapata com as do pilar, é possível resolver o problema dado na Equação 4, o qual permite determinar as dimensões $B_{1}$ igual a $310 \mathrm{~cm}$ e $B_{2}$ igual $260 \mathrm{~cm}$ (dimensões da sapata, de acordo com a Figura 4).

$$
\begin{gathered}
N_{s d}=1,4\left(G_{k}+Q_{k}\right)=1,4(1.320+570)=2.646 \mathrm{kN} \\
\mathrm{A}=\frac{\left(\gamma_{f}\left(N_{s k}+0,1 G_{k}\right)\right.}{R_{d, s o l o}}=\frac{1,4(1.320+570+0,1 \times 1.320)}{357}=7,93 \mathrm{~m}^{2}
\end{gathered}
$$

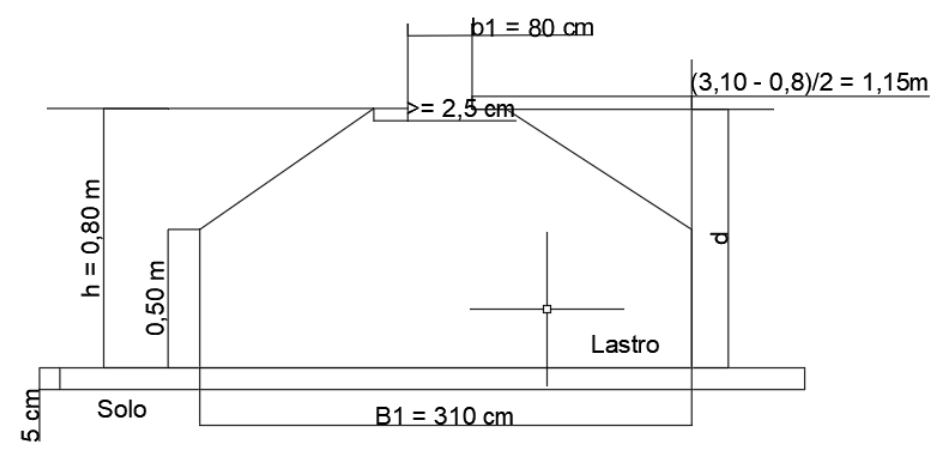

Figura 5: Dimensões da sapata dimensionada. 
Seguindo com o dimensionamento, determinou-se as demais dimensões, como altura total da sapata, altura do rodapé e cobrimento, as dimensões foram verificadas de acordo com a NBR 6122/2010 (ABNT, 2010). Na Figura 5 é possível ver as dimensões encontradas, por meio da representação de um corte frontal da sapata, considerando o lado $B_{l}$.

Nas equações 5 e 6 são apresentados os cálculos para a determinação da área de aço necessária. Na Figura 6 é apresentada uma representação gráfica da disposição do aço na sapata com a quantidade de barras utilizadas em cada direção e os seus respectivos espaçamentos. Na Tabela 2 é apresentado um resumo da disposição das armaduras.

$$
\begin{gathered}
R_{s d 1} \cong R_{s d 2}=\frac{N_{s d}\left(B_{2}-b_{2}\right)}{8_{d}}=\frac{2.646(2,60-0,3)}{8 \times 0,75}=1.014,3 \mathrm{kN} \\
A_{s d 1}=A_{s d 2}=\frac{R_{s d 1}}{f_{y d}}=\frac{1.014,3}{\left(\frac{50}{1,15}\right)}=23,33 \mathrm{~cm}^{2}
\end{gathered}
$$

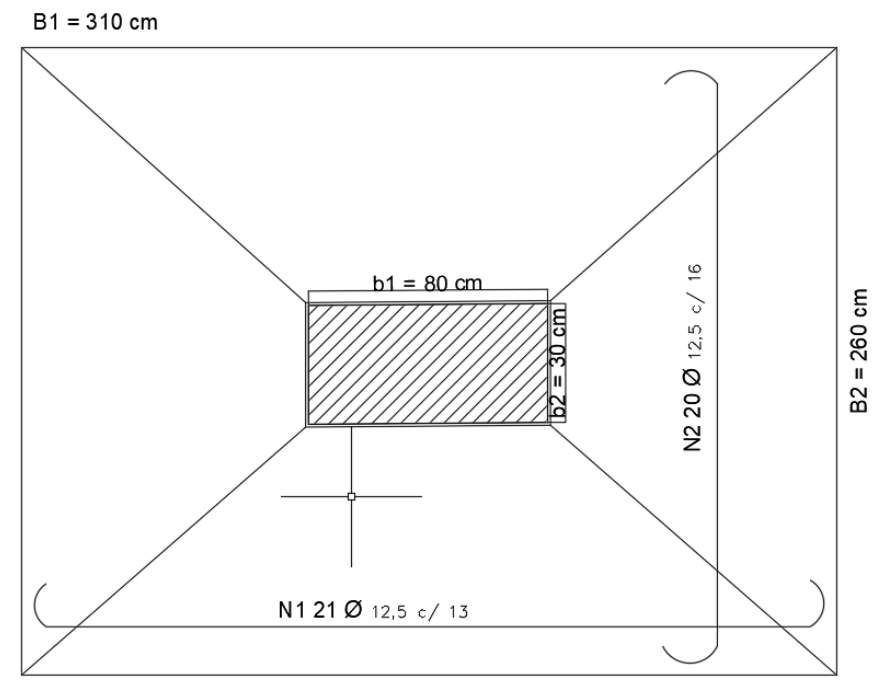

Armaduras em planta

Figura 6: Detalhamento da sapata.

Tabela 2 - Resumo de aço CA - 50 adotado na sapata.

\begin{tabular}{c|c|c|c|c|c|c|c|c|c}
\hline Aço & $\mathrm{N}$ & Diâm. $(\mathrm{mm})$ & Quant. & \multicolumn{2}{c|}{ Comprimento $(\mathrm{cm})$} & Diâm. $(\mathrm{mm})$ & Compr. $(\mathrm{m})$ & Peso $(\mathrm{kg} / \mathrm{m})$ & Total \\
\hline \multirow{2}{*}{ CA-50 } & 1 & 12,5 & 21 & 330 & 6930 & 12,5 & 130,90 & 0,963 & 126.06 \\
\cline { 2 - 9 } & 2 & 12,5 & 20 & 280 & 6160 & 12,5 & 130,90 & 0,963 & 126.06 \\
\hline
\end{tabular}

Por fim, relata-se que foram realizadas todas as verificações recomendadas pela NBR 6122/2010 (ABNT, 2010), a verificação da ruptura por compressão diagonal e de ruptura por punção.

\subsection{Descrição do modelo implementado para análise da despassivação do aço}

Para a determinação da profundidade de carbonatação ao longo do tempo, e consequentemente, do tempo de despassivação do aço, foi utilizada a formulação analítica proposta por Possan (2010), que é apresentada na Equação 7. Alguns dos parâmetros da formulação são obtidos em função das características do concreto analisado e do ambiente ao qual está inserido, outros parâmetros são coletados das Tabelas 3.a e 3.b.

$$
y=k_{c}\left(\frac{20}{f_{c}}\right)^{k_{f c}} \cdot\left(\frac{t}{20}\right)^{\frac{1}{2}} \cdot \exp \left[\left(\frac{k_{a d} \cdot a d^{\frac{3}{2}}}{40+f_{c}}\right)+\left(\frac{k_{c o_{2}} \cdot C O_{2}}{60+f_{c}}\right)-\left(\frac{k_{R U} \cdot(U R-0,58)^{2}}{100+f_{c}}\right)\right] \cdot k_{c e} \quad \text { (Equação 7) }
$$

Onde:

$\mathrm{y}=$ profundidade de carbonatação média do concreto, em $\mathrm{mm}$;

$\mathrm{f}_{\mathrm{c}}=$ resistência característica à compressão axial do concreto, em $\mathrm{MPa}$;

$\mathrm{k}_{\mathrm{c}}=$ fator variável referente ao tipo de cimento empregado (Tabela 3.a);

$\mathrm{k}_{\mathrm{fc}}=$ fator variável referente à resistência à compressão axial do concreto, em função do tipo de cimento utilizado (Tabela 3.a); 
$\mathrm{t}=$ idade do concreto, em anos;

$\mathrm{a}_{\mathrm{d}}=$ teor de adição pozolânica no concreto, em \% em relação à massa de cimento;

$\mathrm{k}_{\mathrm{ad}}=$ fator variável referente às adições pozolânicas do concreto - sílica ativa, metacaulim e cinza de casca de arroz, em função do tipo de cimento utilizado (Tabela 3.a).

$\mathrm{UR}=$ umidade relativa média, em $\% * 0,01$;

$\mathrm{K}_{\mathrm{RU}}=$ fator variável referente à umidade relativa, em função do tipo de cimento utilizado (Tabela 3.a)

$\mathrm{CO}_{2}=$ teor de $\mathrm{CO}_{2}$ da atmosfera, em $\%$.

$\mathrm{k}_{\mathrm{co} 2}$ = fator variável referente ao teor de $\mathrm{CO}_{2}$ do ambiente, em função do tipo de cimento utilizado (Tabela 3.a)

$\mathrm{k}_{\mathrm{ce}}=$ fator variável referente à exposição à chuva, em função das condições de exposição da estrutura (Tabela 3.b).

Tabela 3 - Coeficientes do modelo em função (a) das características do concreto e das condições ambientais (b) das condições de exposição (POSSAN, 2010).

a)

\begin{tabular}{c|c|c|c|c|c}
\hline \multirow{2}{*}{$\begin{array}{c}\text { Tipo de } \\
\text { Cimento }\end{array}$} & \multicolumn{2}{|c|}{ Características do concreto } & \multicolumn{2}{c}{$\begin{array}{c}\text { Condições } \\
\text { Ambientais }\end{array}$} \\
\cline { 2 - 6 } & Cimento & $\mathrm{f}_{\mathrm{c}}$ & Adição & $\mathrm{CO}_{2}$ & $\mathrm{UR}$ \\
\cline { 2 - 6 } & $\mathrm{k}_{\mathrm{c}}$ & $\mathrm{k}_{\mathrm{fc}}$ & $\mathrm{k}_{\mathrm{ad}}$ & $\mathrm{k}_{\mathrm{CO} 2}$ & $\mathrm{k}_{\mathrm{UR}}$ \\
\hline CP I & 19,80 & 1,70 & 0,24 & 18,00 & 1300 \\
\hline CP II E & 22,48 & 1,50 & 0,32 & 15,50 & 1300 \\
\hline CP II F & 21,68 & 1,50 & 0,24 & 18,00 & 1100 \\
\hline CP II Z & 23,66 & 1,50 & 0,32 & 15,50 & 1300 \\
\hline CP III & 30,50 & 1,70 & 0,32 & 15,50 & 1300 \\
\hline CP IV & 33,27 & 1,70 & 0,32 & 15,50 & 1000 \\
\hline CP V ARI & 19,80 & 1,70 & 0,24 & 18,00 & 1300 \\
\hline
\end{tabular}

b)

\begin{tabular}{l|c}
\hline \multicolumn{2}{c}{$\begin{array}{c}\text { Condições de exposição da } \\
\text { estrutura }\end{array}$} \\
\hline \multicolumn{1}{c}{ Proteção à chuva } & $k_{c e}$ \\
\hline $\begin{array}{l}\text { Ambiente interno Protegido } \\
\text { da chuva }\end{array}$ & 1,30 \\
\hline $\begin{array}{l}\text { Ambiente externo } \\
\text { Protegido da chuva }\end{array}$ & 1,00 \\
\hline $\begin{array}{l}\text { Ambiente externo } \\
\text { Desprotegido da chuva }\end{array}$ & 0,65 \\
\hline
\end{tabular}

\subsection{Descrição do modelo implementado para perda de seção do aço}

Como estamos interessados em analisar sapatas de concreto susceptíveis à corrosão uniforme, o diâmetro da armadura, $\phi$, sofre um redução ao longo do tempo, sendo necessário determiná-la a cada instante de análise. Dessa forma, foram utilizadas formulações que fornecessem o diâmetro integro de aço (STEWART; ROSOWSKY, 1998) e a taxa de corrosão (VU; STEWART, 2000) ao longo do tempo, dadas respectivamente nas Equações (8) e (9).

$$
\begin{gathered}
\phi\left(t_{p}\right)=\phi_{0}-\left(2 \cdot 0,0116 \cdot \eta \cdot t_{p}\right) \\
\eta=\left(\frac{37,8 \cdot(1-a / c)^{-1,64}}{c o b}\right) \cdot 0,85 \cdot\left(t_{p}\right)^{-0,29}
\end{gathered}
$$

em que $\phi\left(t_{p}\right)$ é o diâmetro integro de aço (em mm) no tempo $t_{p}$ (em anos) após iniciar o período de progressão da corrosão, calculado por (4), $\phi_{0}$ é o diâmetro inicial da barra $(\mathrm{mm}), \eta$ representa a taxa de corrosão da armadura $\left(\mu \mathrm{A} / \mathrm{cm}^{2}\right), a / c$ é a relação água/cimento do concreto e $c o b$ é a espessura do concreto de cobrimento ( $\mathrm{mm})$.

\subsection{Simulação numérico-probabilística via Monte Carlo}

A fim de analisar probabilisticamente a falha de sapatas de concreto armado no decorrer de sua vida útil de projeto, as formulações anteriormente descritas, foram acopladas e calculadas via teoria da confiabilidade. As modelagens e análises foram realizadas por meio da simulação de Monte Carlo.

O método de simulação de Monte Carlo é um procedimento numérico, amplamente utilizado em diversas áreas, para a realização de experimentos aleatórios em problemas de confiabilidade. Neste método, amostras de variáveis aleatórias são utilizadas para construir um conjunto de realizações do experimento com o intuito de descrever a falha, os espaços seguros, correlacionados à confiabilidade, e calcular a probabilidade do evento, conforme Equação 10.

As amostras utilizadas para o cálculo da probabilidade de falha, são construídas com base na distribuição estatística atribuída a cada variável aleatória do problema. Como o método está baseado na simulação da função de estado limite, quanto maior for a amostra gerada, mais precisa será a descrição do espaço e mais precisa será a probabilidade de falha ou confiabilidade determinada (BECK, 2018). 


$$
P_{f}=\int_{G \leq 0} f_{x}\left(x_{1}, x_{2}, \ldots, x_{n}\right) d x_{1}, d x_{2}, \ldots, d x_{n}
$$

(Equação 10)

em que $f_{x}(X)$ é a função conjunta de densidade das variáveis $X$.

Como a avaliação da integral definida na Equação 10 é quase impossível na prática, procedimentos alternativos foram desenvolvidos com base no conceito de índice de confiabilidade $\beta$ (BECK, 2018). O índice de confiabilidade é definido como a distância entre o ponto médio e o ponto de falha alocado sobre a função de estado limite, $G(X)=0$.

De forma geral, o método consiste na construção de uma amostra para as variáveis aleatórias envolvidas no problema a ser simulado, e assim, a probabilidade de falha é calculada utilizando um estimador, baseado na avaliação da função de estado limite, de acordo como apresentado na Equação 11. O estimador $I\left(x_{i}\right)$, baseado na função de estado limite, é dado em Equação 12, sendo seu valor 0 ou 1, sendo 0 para o caso em que não há falha e, 1, para quando há falha.

$$
\begin{gathered}
P_{f}=\int_{G \leq 0} f_{x}\left(x_{i}\right) d x_{i}=\int_{G \leq 0} I\left(x_{i}\right) f_{x}\left(x_{i}\right) d x_{i}=E\left[I\left(x_{i}\right)\right] \\
I\left(x_{i}\right)= \begin{cases}1, & G \leq 0 \\
0, & G>0\end{cases}
\end{gathered}
$$

Simulando a função de estado limite para um número conveniente de amostras, o valor médio $I\left(x_{i}\right)$ será uma estimativa para a probabilidade de falha, assim, de acordo com a Equação 13 a probabilidade de falha pode ser facilmente estimada para todo o conjunto de amostras.

$$
\overline{P_{f}}=E\left[I\left(x_{i}\right)\right]=\frac{1}{N} \sum_{i=1}^{N} I\left(x_{i}\right)
$$

em que $N$ é o número de simulações, isto é, de avaliações da equação de estado limite do problema analisado.

Definido o método de estimativa da probabilidade de falha, a equação de estado limite a ser analisada neste trabalho é dada na Equação 14 e, refere-se ao evento da sapata falhar durante a vida útil de projeto $\left(\mathrm{g}_{1}\right)$ ou o solo entrar em colapso $\left(\mathrm{g}_{2}\right)$.

$$
g(x)=\min \left(g_{1}, g_{2}\right) \text { onde }\left\{\begin{array}{c}
g_{1}(x)=\sigma_{t r}-\sigma_{t s} \\
g_{2}(x)=\sigma_{a d m}-\sigma_{s t}
\end{array}\right.
$$

em que $\sigma_{t r}$ é a tensão de tração resistida pelo elemento de fundação, $\sigma_{s r}$ é a tensão de tração solicitada pela sapata, $\sigma_{a d m}$ é a tensão admissível do solo, e $\sigma_{s t}$ é a tensão transmitida pelo elemento de fundação. A determinação das tensões foi realizada de acordo com as formulações estabelecidas pelo método de dimensionamento de bielas e tirantes e pelos métodos de estimativa da capacidade de carga de Vésic (1974) e Terzaghi (1943).

Assim, de acordo com a expressão apresentada na Equação 14, tem-se que a falha do evento ocorre quando $g \leq 0$, e não falha quando $g>0$. Observe, que neste trabalho, como $g$ é uma função que adota o mínimo de duas outras funções $\left(\mathrm{g}_{1} \mathrm{e}\right.$ $\mathrm{g}_{2}$ ), a falha é identificada quando ocorre falha no elemento estrutural ou no solo.

Tabela 4 - Variáveis aleatórias.

\begin{tabular}{c|c|c|c}
\hline Parâmetros & média & $\begin{array}{c}\text { Coeficiente } \\
\text { de variação }\end{array}$ & Distribuição \\
\hline $\mathrm{NSPT}$ & 20 & $15 \%$ & Normal \\
\hline $\mathrm{f}_{\mathrm{ck}}(\mathrm{MPa})$ & 12,5 & $10 \%$ & Normal \\
\hline $\mathrm{cob}(\mathrm{cm})$ & 5 & $15 \%$ & Normal \\
\hline $\mathrm{UR}(\%)$ & 50 & $20 \%$ & Log-Normal \\
\hline$\eta\left(\mu \mathrm{A} / \mathrm{cm}^{2}\right)$ & 10 & $30 \%$ & Normal \\
\hline
\end{tabular}

Por fim, na Tabela 4 são apresentadas as variáveis aleatórias que foram utilizadas nas simulações. Comenta-se que os valores foram definidos após analisar alguns trabalhos da literatura. Todas as demais variáveis, não apresentadas na Tabela 4, foram tratadas neste trabalho como determinísticas. 


\section{RESULTADOS}

Inicialmente, na Figura 7 é apresentado o avanço da profundidade de carbonatação do concreto, principal agente agressivo considerado na despassivação do aço das sapatas de concreto e, por consequência, a perda de seção útil de aço, ou seja, a perda de aço que resiste aos esforços de tração na sapata. Os resultados foram obtidos considerando os valores médios das variáveis aleatórias, conforme Tabela 4, e fazendo o uso das Equações 7, 8 e 9.

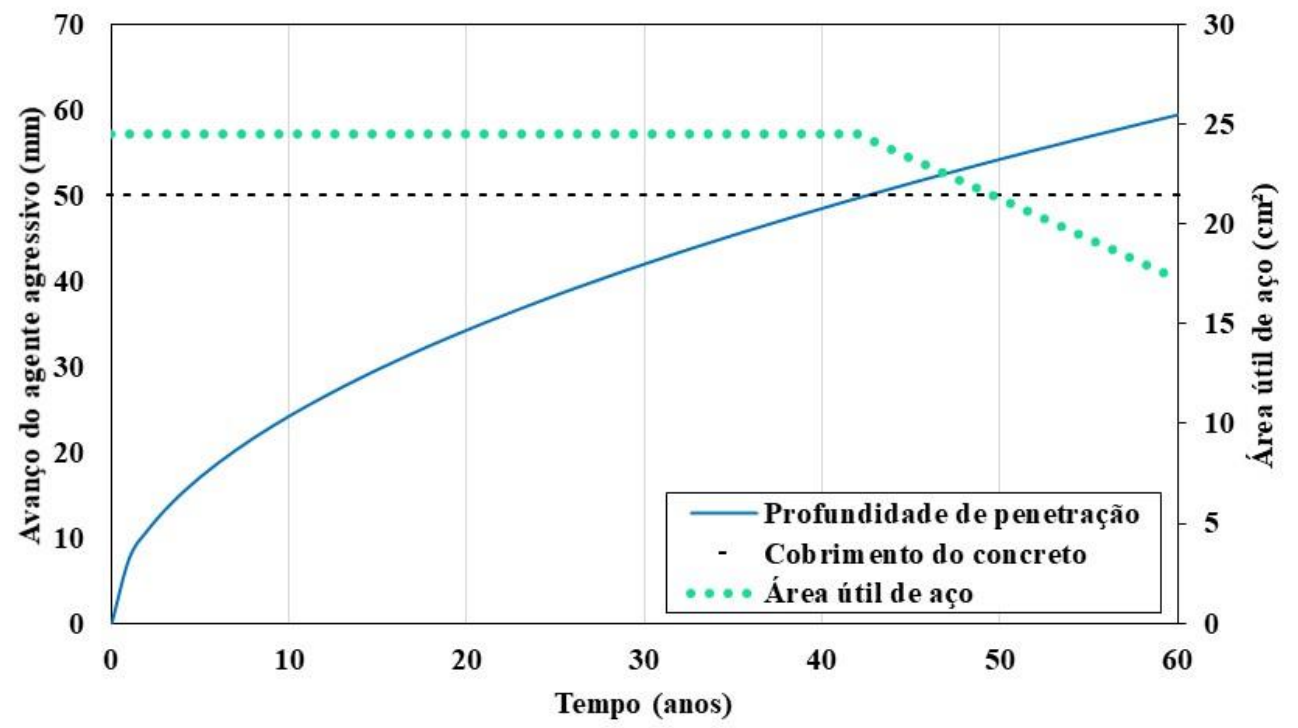

Figura 7: Avanço da penetração dos agentes agressivos e da perda de aço resistente.

Observa-se na Figura 7 que durante a fase de iniciação da corrosão a área útil de aço das armaduras não é alterada, de acordo com a Equação 9, e que, a partir do momento em que ocorre a despassivação da armadura, 42 anos, a perda de seção acontece de forma continua e linear, diante da consideração que nenhuma manutenção ou reparo é realizado no elemento estrutural, conforme observado por Vu e Stewart (2000).

Vu e Stewart (2000) observaram por meio de experimentos que a perda de seção de aço ocorre com maior intensidade nos primeiros anos da manifestação patológica, e que, se o processo de degradação não é cessado, a perda de seção útil de aço pode chegar a 50\% em 10 anos. Para a sapata analisada, verificou-se que a redução de área útil de aço foi de 29,73 $\%$, sendo a área média inicial de $24,54 \mathrm{~cm}^{2}$, e após 60 anos de exposição aos agentes agressivos, de $17,24 \mathrm{~cm}^{2}$. Para esta redução, observou-se que tanto o elemento estrutural, entraria em ruína, quanto o solo, já que são verificadas mudanças significativas na configuração geométrica da fundação, o que levaria a uma ruptura generalizada do solo.

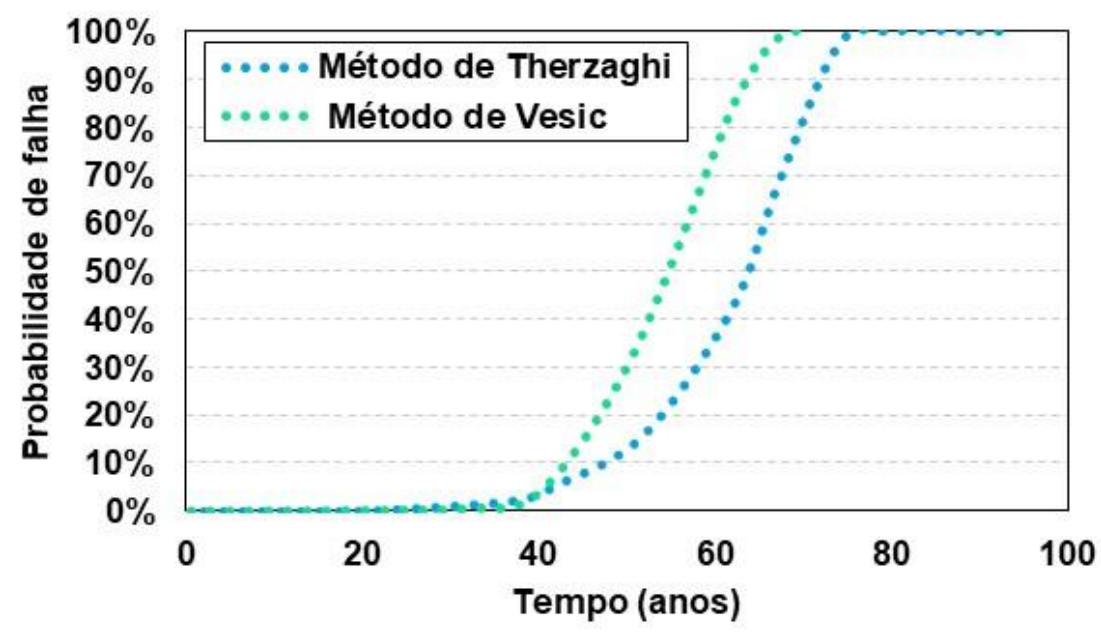

Figura 8: Probabilidade de falha ao longo do tempo. 
De forma geral, observou-se que reduções acima de $20 \%$ da área resistente de aço, levam a ruína da sapata de concreto, e mais, que se o método de análise de falha não considerasse a perda de capacidade mecânica do elemento estrutural, a falha seria definida pela perda de capacidade de carga, independente do tipo de formulação adotada, Vesic (1974) ou Terzaghi (1943).

A partir dos resultados apresentados na Figura 8, verificou-se que a probabilidade de falha da sapata, que neste trabalho refere-se ao elemento entrar em colapso seja pela falta de capacidade de carga do solo ou pela perda de resistência mecânica do elemento estrutural, é nula para os 28 primeiros anos de vida, independentemente do tipo de formulação utilizada para estimativa da capacidade de carga, resistência do solo (considerando a faixa de variação do NSPT), da umidade relativa do meio e da resistência à compressão do concreto.

Das análises realizadas, observou-se que para as faixas de variação dos parâmetros aleatórios estudados, a falha geral dada pelo mínimo entre as funções de falha $\mathrm{g}_{1}$ e $\mathrm{g}_{2}$ (Equação 14), ocorre inicialmente (considerando a progressão da corrosão) devido ao colapso do solo $\left(\mathrm{g}_{2}<0\right)$ e depois para a ruína da estrutura concomitantemente à do solo $\left(\mathrm{g}_{1}<0\right.$ e $\left.\mathrm{g}_{2}<0\right)$. Desta forma, não foram identificados casos onde ocorresse a ruína do elemento de concreto sem o colapso do solo. Mostrando que os parâmetros de seguranças adotados no dimensionamento do elemento estrutural, ainda sobressaem aos das formulações de análise da capacidade do solo e da iteração solo-estrutura.

Ademais, acrescenta-se que a partir do momento em que ocorre a despassivação do aço, as probabilidades de falha aumentam drasticamente, independente da formulação empregada, e que a formulação de Vésic (1974) gerou probabilidades de falha superiores à de Terzaghi (1943). Ainda, relata-se que independente do método empregado para a determinação da capacidade de carga, a chance de a sapata entrar em colapso antes dos 60 anos, é grande, apresentando uma probabilidade média de $58 \%$.

\section{CONCLUSÕES}

Diante dos resultados obtidos com as análises determinísticas e probabilísticas, pode-se concluir que:

- Um cobrimento adequado garante não só um período maior para levar à despassivação da armadura como também para a sapata perder sua capacidade mecânica ao ponto de levar a falha do elemento de concreto;

- Concretos produzidos com cimentos de maior durabilidade, por exemplo, CP V, seriam recomendados para a produção de sapatas em solos corrosivos, já que proporcionariam maior dificuldade de penetração dos agentes agressivos, levando nestes casos a índices de confiabilidade maiores (menores probabilidades de atingir a falha);

- Diante das análises, observou-se que das quatro variáveis aleatórias utilizadas, o NSPT e o $\eta$, foram as que apresentaram maior relevância e sensibilidade frente à probabilidade de a sapata atingir o falha durante sua vida útil.

Desta forma, diante dos resultados obtidos, a durabilidade de sapatas de concreto armado deve ser associada a valores mais rigorosos de espessura de cobrimento, bem como concretos menos permeáveis e uma melhor análise do futuro ambiente de exposição para que um correto tipo de cimento seja adotado e potencialize a durabilidade do componente estrutural.

Acredita-se, ainda, que a abordagem probabilística do problema da vida útil através da consideração da aleatoriedade das variáveis envolvidas demonstra-se adequada para uma determinação mais realística da capacidade mecânica de elementos de fundação e do solo. Ademais, os resultados apontam a aplicabilidade da metodologia adotada para tratar a aleatoriedade das variáveis de problemas correlatos à área de patologia das construções.

\section{REFERÊNCIAS}

ABMS/ABEF. Fundações teoria e prática. 3 a edição, São Paulo: PINI, p. 802, 2016.

ANDRADE, C. Manual para diagnóstico de obras deterioradas por corrosão de armaduras. Trad. De Antonio Carmona e Paulo Helene. São Paulo, Pini, 1992.

ASSOCIAÇÃO BRASILEIRA DE NORMAS TÉCNICAS. NBR 6118: Projeto de estruturas de concreto. Rio de Janeiro, 2014. 
ASSOCIAÇÃO BRASILEIRA DE NORMAS TÉCNICAS. NBR 6122: Projeto e execução de fundações. Rio de Janeiro, 2010.

BECK, A. T. Confiabilidade e segurança das estruturas. São Paulo: Elsevier Campus, p. 448, 2019.

CAMPOS, J. C. Elementos de fundação de concreto. São Paulo: Oficina de Textos, p. 544, 2015.

CARMONA, A. F.; MAREGA, A. Retrospectiva da patologia no Brasil: Estudo Estatístico, in: Jornadas em Español y Portugués sobre Estructuras y Materiales, CEDEX, IETcc, (1988), pp. 325-348, 1988.

CINTRA, J. C. A.; AOKI, N.; ALBIERO, J. H. Tensão admissível em fundações diretas. São Carlos: Rima, 2003.

DAL MOLIN, D. C. C. Fissuras em estruturas de concreto armado: análise das manifestações típicas e levantamento de casos ocorridos no Estado do Rio Grande do Sul. Dissertação de Mestrado em Engenharia, Universidade Federal do Rio Grande do Sul, Porto Alegre, 1988.

GEIKER, M. R.; POLDER, R. B. Experimental support for new electro active repair method for reinforced concrete, Materials and Corrosion, V.67, pp. 600-606, 2016.

LONGEAIS, L. La pathologie des foundations. Paris: Edition du Moniteur, 1982.

MEHTA, P. K.; MONTEIRO, P. J. M. Concreto. Microestrutura, propriedades e materiais. 4. ed. São Paulo: IBRACON, 2014.

POSSAN, E. Modelagem da carbonatação e previsão de vida útil de estruturas de concreto em meio urbano, Tese de Doutorado em Engenharia, Programa de Pós-Graduação em Engenharia Civil, Universidade Federal do Rio Grande do Sul, Porto Alegre, 2010.

SILVA JUNIOR, F. C. Uma revisão sobre manifestações patólogicas mais frequentes em fundações de concreto de edificações. Trabalho de conclusão de curso, Universidade Estadual de Feira de Santana, Bahia, 2008.

VU, K. A. T.; STEWART, M. G. Structural reliability of concrete bridges including improved chloride-induced corrosion models. Structural Safety, 22:313-333, 2000.

VELLOSO, D. A.; LOPES, F. R. Fundações: critérios de projetos, investigação do subsolo, fundações superficiais e fundações profundas. São Paulo: Oficina de Textos, p. 568, 2010.

VU, K. A. T.; STEWART, M. G. Structural reliability of concrete bridges including improved chloride-induced corrosion models. Structural Safety, 22:313-333, 2000. 